\title{
On the Practice and Experience of Anti-peccancy Work in Electricity Production
}

\author{
Zhang Da, Zhao Qingyuan, Li Ming \\ Benxi Power Supply Company of National Grid Liaoning Electric Power Co., Ltd. \\ bxwl_650928@126.com
}

Keywords: Anti-peccancy; electricity enterprises

\begin{abstract}
What is habitual peccancy? It happens when workers adhere to incorrect practices and habits that are not in accordance with correct operating methods or safety regulations. In this paper, the author offers an in-depth and accurate analysis on the causes of habitual peccancies and proposes countermeasures to prevent and reduce effectively accidents, beneficial for the improvement of safety production management level in electricity industry and the creation of a safe and harmonious working environment.
\end{abstract}

Production safety anti-peccancy requires enterprises to cherish lives of their own and others. They do not violate regulations and should also correct other's acts of peccancy promptly if there is any, which is in fact a preventive measure to avoid production safety accidents from the source. As shown in the past experiences of production safety work, over $95 \%$ of production safety accidents in electricity enterprises are caused by peccancies (administrative peccancy, habitual peccancy, directional peccancy, workability peccancy, equipment peccancy and joint result of various peccancies). Therefore, in order to prevent effectively production safety accidents, it is necessary to curb peccancies. This has been recognized by enterprises' leaders, safety supervision staff and teams of workers. As some workers said: "production safety anti-peccancy" activities fully embody the essential people-oriented and life-cherishing requirements, as they are based on the true emotion of caring themselves and others and at the same time are the only way to promote safety culture in enterprises.

\section{Present Status of Anti-peccancy Work in Electricity Enterprises}

As a comprehensive work, one of the major risks of security work is peccancy, including management peccancy, equipment peccancy, conducts peccancy and so on. In recent years, electricity enterprises have carried out widely anti-peccancy activities and achieved certain effects. For workers, these activities help protect their life security and the happiness of their families; for enterprises, they guarantee the sound operation of electricity devices and improve the sound level of equipment, thus forming a solid foundation for the enterprises to fulfill their social responsibilities, provide quality services and establish a positive corporate brand image and social reputation.

\section{Analyzing the Causes of Inefficient Performance in the Anti-peccancy Work of Electricity Enterprises}

As can be seen in the present status of anti-peccancy work, the enterprises have achieved certain effects, but still far from the requirements of safety management, the "controllable, controllability and in control" prospect of safety production and zero peccancy objective. The author believes that factors that affect the effectiveness of anti-peccancy work are mainly the following aspects:

\section{Influence of Negative Environment on the Workers' Behavior.}

As the saying "who keeps company with the wolf will learn to howl" clarifies the influence of environment, including soft environment and hard environment. The environment can affect a person's mental attitude directly, showing as changes in behaviors. Negative environment will 
contribute to the development of defects in people's characteristics, giving unbeneficial hints, encouraging bad attitudes and inducing unsafe behaviors out of the workers.

Soft environment, namely the cultural environment, is not ignorable as a factor for individuals. In work, workers never exist as individuals but are linked a collective team. Both the big environment of enterprise safety management and the small environment of team safety operation affect directly safety value orientations and behavior habits of the individuals.

Hard environment, namely the operation environment and safety protection equipment, is the basic condition to ensure safety operation of the workers. There is often such a phenomenon in real work that workers no longer pay enough attention to construction process due to the time limit and only highlight the core equipment rather than imply strict standards on auxiliary facilities like safety equipment when checking before acceptance. Thus they ignore that though the construction is finished on schedule, safety of workers operation environment has not been guaranteed and sometimes workers even get implications of lowering work standards freely.

Cultural environment cannot be created overnight but a result of accumulation and precipitation of long-term practices. The key of establishing soft environment lies in all levels of managers and team leaders, whose long-term violations of regulations and even leading role in peccancy would promote the formation of negative environment. Unconsciously influenced by such an environment, members of the team, especially those new comers would follow suit and get accustomed to acts of violations and peccancies, leading to the gradual degradation of identifying ability and vigilance against peccancies

\section{Influence of Management Deficiencies on Anti-peccancy Work.}

Anti-peccancy working mechanism has some limitations. The existing anti-peccancy working mechanism in electricity enterprises generally contains two modes: behavior control and regular report of subordinate units. Inspection and correcting peccancies are generally unidirectional and mainly depend on the one-way inspection and correction from the upper levels to the subordinate levels that are unexpected inspections and judgments by individuals on certain scene. Such a working mechanism has certain limitations. The first one is that there is phenomenon of evading the crucial points and doing work carelessly, or deducting fines from bonuses that makes the fine-taking cycle expend to monthly or yearly, leading to failure in achieving the designed discipline and moral educational effect. The second is that peccancy education only focuses on economic fining and fails to make the workers reflect and re-understand the situation in depth. The third is insufficient regulation of the leaders. The leaders lack efficient inspection and regulation measures. All the above show that the accountability of anti-peccancy work has not been thoroughly investigated. Therefore, the author believes that it is necessary to deepen further the focus of anti-peccancy work. The fourth is that the peccancy levels during inspection and correction process of safety production differ within workers and thus are restricted. The fifth is the ranges of peccancy during inspection and correction process of safety production are restricted to some extent.

\section{Causes of Peccancy in Safety Production}

\section{Ideological aspect of workers.}

(1)The workers are afraid of assuming responsibilities and believe in "do more and being punished more, do less and being punished less". This fault ideology of them has seriously affected their job. Is everyone can pay a little more carefulness on the work; there would be less unsafe factors and less possibility of an accident.

(2)The workers lack sense of responsibility. They are not careful enough when working and even follow the majority or loaf on their job. These irresponsible statuses are generally the main cause for peccancy.

(3)The workers want to take chances and subjectively believe that there would not be any negative consequences, though they know that it violates the safety regulations. For example, the spot workers often need to inspect from the main control room to the high-pressure room; in the former the workers are not required to wear safety helmet but are in the later. However, some 
workers assumes that nothing would happen if they enter the high-pressure room while wearing no helmet and their thoughts get paralyzed, thus leading to the phenomenon of peccancy.

(4)The workers are weak in the crisis consciousness and believe that peccancy cannot be equaled to accident but rather a way to save time and labor. For example, bar workers see that there is no one on the ground during construction and thus throw a bolt after informing the guardian. He tells others confidently that why not since it could not injure any person or equipment but save time and effort. However, he does not realize the habitual peccancy would be formed if he continues to throw a bolt today and a golden toll tomorrow.

\section{Quality and Abilities of Operating Individuals.}

(1)Peccancy behaviors are easily to occur as the structure of workers in large-scale construction is complicated and their skills also differ with each other. For example, as in large-sale line construction, the construction points are large in number, wide in scale, of long distance and involve many manpower and material resources in outdoor work, which makes it easier for construction workers following regulations to organize joint peccancies.

(2)Migrant workers hired temporarily during construction have a high mobility, lack constrictions and in addition have a low educational level and have received little safety knowledge, which make them more easily to act peccancies. Take such a simple regulation as wearing safety helmet as example. No matter how times being educated, there are always some workers who wear off or do not wear appropriate consciously or unconsciously the helmet during work.

(3)Due to limited level of skills and changes of job, new workers are more likely to come across the boundary of regulations and commit peccancies. For example, skill-intensive fields like automation systems with a wide range of skills and quick equipment updating pace require the workers to have a wide range of knowledge, a high skill level and a strong ability to analyze and solve problems. However, for new workers who just entered to the plant are unfamiliar with the equipment and working process and do not have enough knowledge of dangerous pints in the work, etc. which make them easier to commit peccancy.

\section{Enterprise Safety Management.}

(1) The enforcement is not enough. Many workers do not receive a fine enough to warn themselves or others or simply do not fear of being inspected due to the loose management of managing personnel even if they are caught with peccancies.

(2) The lack of rigid standardized requirements. Due to the large job flexibility and mobility, the enterprises are often facing with problems beyond the listed safety range, resulting in unsatisfying performance of the team in standardized operation and enforcing working procedures.

(3) The enforcement of standardization is not enough. The standardization is not implemented well into practice: the site survey is not careful enough, leading to inadequate survey records or incomplete safety measures; the working procedure has changed and the designed standardization list is no longer suitable.

(4) The workers do not follow arrangements of the leaders and work according to their own thoughts and labor distribution in order to keep up with the time limit, regardless of the pre-team meetings and safety explanations.

\section{Conclusion}

In conclusion, the range of safety regulation should cover the main business, multi-operation, rural electricity, infrastructure and other aspects, so as to ensure the definite safety of production by detecting abnormal conditions in time and correcting and stopping peccancies promptly.

\section{References}

[1] Eighteen Major Grid Anti-accident Measures for the National Power Grid Corporation [Z].

[2] Construction Project Management [Z].

[3] Compiled Provisions for National Grid Construction Site Safety Management [Z]. 
[4] Tian Yuping. Anti-peccancy Question and Answer in Power Enterprise [M]. Beijing: China Electric Power Press, 2008.

[5] Li Donglai, Zhu Jianjun. Law and Prevention of Peccancies[J]. China Power Enterprise Management, 2010.

[6] Huang Xianhua. On the Application of Anti-peccancy in Power Production Safety[J]. China Electric Power Education, 2009. 